\title{
Rabi Regime of Current Rectification in Solids
}

\author{
Oles Matsyshyn $\odot,{ }^{1}$ Francesco Piazza, ${ }^{1}$ Roderich Moessner, ${ }^{1}$ and Inti Sodemann ${ }^{1,2,3}$ \\ ${ }^{1}$ Max Planck Institute for the Physics of Complex Systems, Dresden 01187, Germany \\ ${ }^{2}$ Department of Physics and Astronomy, University of California, Irvine, California 92697, USA \\ ${ }^{3}$ Institut für Theoretische Physik, Universität Leipzig, D-04103 Leipzig, Germany
}

(Received 15 April 2021; revised 29 June 2021; accepted 26 August 2021; published 17 September 2021)

\begin{abstract}
We investigate rectified currents in response to oscillating electric fields in systems lacking inversion and time-reversal symmetries. These currents, in second-order perturbation theory, are inversely proportional to the relaxation rate, and, therefore, naively diverge in the ideal clean limit. Employing a combination of the nonequilibrium Green function technique and Floquet theory, we show that this is an artifact of perturbation theory, and that there is a well-defined periodic steady state akin to Rabi oscillations leading to finite rectified currents in the limit of weak coupling to a thermal bath. In this Rabi regime the rectified current scales as the square root of the radiation intensity, in contrast with the linear scaling of the perturbative regime, allowing us to readily diagnose it in experiments. More generally, our description provides a smooth interpolation from the ideal periodic Gibbs ensemble describing the Rabi oscillations of a closed system to the perturbative regime of rapid relaxation due to strong coupling to a thermal bath.
\end{abstract}

DOI: 10.1103/PhysRevLett.127.126604

Introduction.-Crystalline solids lacking inversion symmetry can display bulk photovoltaic effects (BPVE) [1-3], namely macroscopic dc rectified currents in response to spatially uniform ac electric fields. There is a long tradition of studying these BPVE [4-9], but also a growing renewed interest in investigating their connections to the Berry phase geometry and topology of electronic bands [10-22], and their potential for novel photovoltaic technologies [10,17,23-27].

Our study is motivated by the following question: what is the ultimate fate of current rectification in Bloch bands in the ideal limit where relaxation times become very large? As we will demonstrate, there is in fact a well-defined periodic steady state in such a limit, that we will refer to as the Rabi regime, in which the system sustains a finite dc rectified current.

A useful starting point to appreciate the nontrivialities of such a clean limit is to analyze the problem perturbatively in the amplitude of electric field, as is commonly done in most studies (see however Refs. [12,28-31]). Perturbation theory predicts a rectified current $\mathbf{j}$, that grows as the square of the amplitude of the field, $\mathbf{j} \propto|\mathbf{E}|^{2}$. For frequencies above the threshold for interband transitions, such perturbative BPVEs are often separated into two mechanisms

Published by the American Physical Society under the terms of the Creative Commons Attribution 4.0 International license. Further distribution of this work must maintain attribution to the author(s) and the published article's title, journal citation, and DOI. Open access publication funded by the Max Planck Society. known as the shift and the injection current effects [3-10, 12-17,23-26,29-31]. The injection current originates from difference of the band-diagonal velocity of the empty and occupied bands at a given crystal momentum $\mathbf{k}$. The shift current, on the other hand, originates from the difference of positions of Bloch wave functions between the empty and occupied bands at a given $\mathbf{k}$, and can be computed as the contribution arising from the band-offdiagonal velocity operator.

A crucial distinction between the shift and injection currents is that, within perturbation theory, the shift current appears to have a finite value in the clean limit of vanishing relaxation rate, $\Gamma \rightarrow 0$, while the injection current appears to diverge in such limit as $1 / \Gamma$, which ultimately arises from the vanishing quasienergy denominators appearing at higher orders of the perturbation theory for the rectified current (see Refs. [3-10,12-17,23-26,29-31] and Supplemental Material, Sec. A [32]). Such divergence is often handled in an ad hoc manner by computing the response of the rate of change of the current, $d \mathbf{j} / d t$, and assuming that such growth leads to a current saturation to a value proportional to the relaxation time $\tau \sim \hbar / \Gamma$. However, recently an interesting nonperturbative study of the circularphotogalvanic effect (CPGE) in Weyl semimetals [30] demonstrated that the rectified current saturates to a finite value even in the clean limit of vanishing relaxations $(\Gamma \rightarrow 0)$ within a semiclassical kinetic framework. The underlying mechanism for such saturation is the Rabi dynamic broadening of absorption [33-36], which occurs when the energy scale controlling the transitions between conduction $(c)$ and valence $(v)$ bands exceeds the relaxation rate, $e \mathbf{E} \cdot\langle c|\mathbf{r}| v\rangle \gg \Gamma$, which we refer to as the Rabi regime. 
In the present Letter we develop a microscopic description of the currents for arbitrary values of the nonlinearity parameter $e \mathbf{E} \cdot\langle c|\mathbf{r}| v\rangle / \Gamma$, that captures the perturbative and the Rabi regimes on equal footing. To do so, we employ a Keldysh-Floquet formalism [37-39] in a generic two-band system coupled to an ideal fermionic bath, following the pioneering approach of Ref. [12] (see also Ref. [28]). As we will see, and contrary to the expectations of perturbation theory, in the Rabi regime, the traditional resonant shift current contributions vanish, whereas the injection currents approach a finite limit that scales as the square root of the radiation intensity in a sharp contrast to perturbation theory expectations. We will also demonstrate that the Rabi regime can be viewed as an example of thermalizing synchronization of a system under an external periodic drive that can be described by the periodic Gibbs ensemble [40,41].

Keldysh-Floquet formalism.-We derive the nonperturbative expression for currents within a two-band model [see Fig. 1(a)]. The electric current operator is defined as: $\hat{\mathbf{j}}=e \hat{\mathbf{v}} / \hbar=\partial \hat{H}_{0}[\mathbf{k}+e \mathbf{A}(t) / \hbar] / \partial \mathbf{A}(t)$, where $\hat{H}_{0}(\mathbf{k})$ is the $2 \times 2$ matrix Bloch Hamiltonian, and $\mathbf{A}(t)$ is the vector potential from a spatially uniform but time dependent electric field. Since the crystal momentum $\mathbf{k}$ is conserved, the problem is equivalent to a collection of independent driven two-level systems. We restrict our analysis to a monochromatic electric field with frequency $\omega$ :

(a)

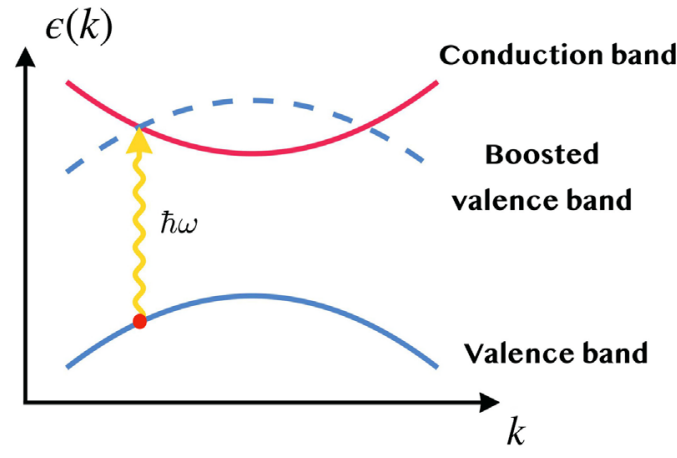

(b)

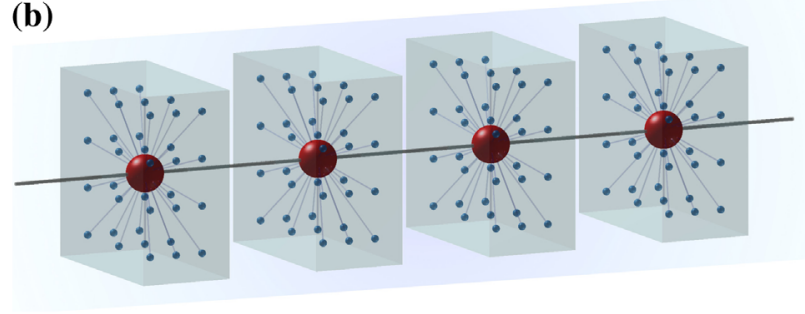

FIG. 1. (a) Energy crossing between boosted valence and conduction bands in Floquet representation, (b) Depiction of underlying tight binding model with physical sites (red balls) which are tunnel coupled (solid lines) among themselves and with their own identical fermionic bath (blue balls).

$$
\mathbf{A}_{0}(t)=i \frac{\mathbf{E}}{\omega} e^{i \omega t}-i \frac{\mathbf{E}^{*}}{\omega} e^{-i \omega t}
$$

Here $\mathbf{E}$ is a vector with complex entries, allowing us to capture light of an arbitrary degree of polarization, ranging from the case of linear polarization, when all components can be chosen to be real, to fully circularly polarized light, when two orthogonal components differ by a phase of $\pi / 2$. The periodicity in time allows us to employ the Floquet picture (for details see Supplemental Material, Sec. B [32]) where multiple Floquet bands appear with a quasienergy that is boosted by multiples of the driving frequency [see Fig. 1(a)]. We simplify the problem by truncating the Floquet Hamiltonian to two bands that are in resonance, in the spirit of a rotating-wave approximation [13]. This approximation is well justified when the off diagonal terms in the Floquet Hamiltonian are smaller in comparison to the Floquet quasienergy difference and to other remote Floquet bands, namely when $e \mid \mathbf{E} \cdot\langle c|\mathbf{r}| v\rangle \ll \hbar \omega$ (see, e.g., Ref. [42] and Supplemental Material, Sec. B [32]). Thus the approximate Floquet Hamiltonian is

$$
\begin{aligned}
H_{\mathbf{F}} & =\left(\begin{array}{cc}
\epsilon_{1}+\hbar \omega & i \frac{e \mathbf{E}}{\hbar \omega} \cdot\left(\frac{\partial H_{0}(\mathbf{k})}{\partial \mathbf{k}}\right)_{12} \\
-i \frac{e \mathbf{E}^{*}}{\hbar \omega} \cdot\left(\frac{\partial H_{0}(\mathbf{k})}{\partial \mathbf{k}}\right)_{21} & \epsilon_{2}
\end{array}\right) \\
& =h_{0}+\mathbf{h} \cdot \boldsymbol{\sigma},
\end{aligned}
$$

where 1 stands for valence, 2 for conduction, and $\epsilon_{1,2}$ are effective valence and conduction band energies respectively (which could be dressed by higher order perturbative corrections with respect to bare band energies, as further discussed in Supplemental Material, Sec. B [32]). The subscript $\mathbf{F}$ stands for the representation of the operator in the Floquet picture, which is related to the ordinary Schrödinger picture as follows:

$$
\hat{O}_{\mathbf{F}}=\left(\begin{array}{ll}
O_{22} & O_{12} \\
O_{21} & O_{11}
\end{array}\right), \quad \hat{O}(t)=\left(\begin{array}{cc}
O_{11} & O_{21} e^{-i \omega t} \\
O_{12} e^{i \omega t} & O_{22}
\end{array}\right) .
$$

In order to capture relaxation processes, we couple the system to a bath and apply the nonequilibrium Green function technique on the Keldysh contour (see Supplemental Material, Sec. C [32] and Refs. [13,37,43-47]). We choose a simple model in which each fermionic site in the system of interest is coupled to its own fermionic bath, with a common hopping amplitude $V_{\text {mix }}$ [see Fig. 1(b)]. The temperature of the bath is $T_{\text {bath }}=1 /\left(k_{B} \beta\right)$ and the chemical potential is $\mu$. The effective density matrix of the system is given by the lesser equal time Green function $G^{<}(t, t)$, and can be shown as follows (see Supplemental Material, Sec. C [32]): 


$$
\begin{aligned}
\hat{\rho}_{\mathbf{F}}= & -i \hat{G}_{\mathbf{F}}^{<}=\left(\begin{array}{cc}
f_{1} & 0 \\
0 & f_{2}
\end{array}\right) \\
& +\frac{f_{1}-f_{2}}{2\left(h^{2}+\frac{\Gamma^{2}}{4}\right)}\left(\begin{array}{cc}
-h_{x}^{2}-h_{y}^{2} & h_{-}\left(h_{z}+i \frac{\Gamma}{2}\right) \\
h_{+}\left(h_{z}-i \frac{\Gamma}{2}\right) & h_{x}^{2}+h_{y}^{2}
\end{array}\right),
\end{aligned}
$$

where $f_{1,2}=1 /\left\{1+\exp \left[-\beta\left(\epsilon_{1,2}-\mu\right)\right]\right\}$ are valence and conduction Fermi-Dirac occupation factors respectively, $h_{ \pm}=h_{x} \pm i h_{y}$ and $\Gamma=2 \pi\left|V_{\text {mix }}\right|^{2}$ is the relaxation rate.

The dc current of the system, $J^{\alpha}=-i e \operatorname{Tr}\left[\hat{G}_{\mathbf{F}}^{<} \hat{v}_{\mathbf{F}}^{\alpha}\right] / \hbar$, can be decomposed into three contributions:

$$
\begin{gathered}
J_{1}^{\alpha}=\frac{e}{\hbar} \int \frac{d \mathbf{k}}{(2 \pi)^{3}} \frac{\frac{\Gamma}{2}\left(f_{1}-f_{2}\right)}{h^{2}+\Gamma^{2} / 4}\left(h_{y} v_{x}^{\alpha}-v_{y}^{\alpha} h_{x}\right), \\
J_{2}^{\alpha}=\frac{e}{\hbar} \int \frac{d \mathbf{k}}{(2 \pi)^{3}} \frac{h_{z}\left(f_{1}-f_{2}\right)}{h^{2}+\Gamma^{2} / 4}\left(h_{x} v_{x}^{\alpha}+h_{y} v_{y}^{\alpha}\right) \\
J_{3}^{\alpha}=-\frac{e}{\hbar} \int \frac{d \mathbf{k}}{(2 \pi)^{3}}\left(f_{1}-f_{2}\right) v_{z}^{\alpha} \frac{h_{x}^{2}+h_{y}^{2}}{h^{2}+\frac{\Gamma^{2}}{4}} .
\end{gathered}
$$

Here $\alpha$ denotes the real space indices and the velocity operator in Floquet representation $\hat{v}_{\mathbf{F}}^{\alpha}$ is decomposed in the Pauli basis, namely $\hat{v}_{\mathbf{F}}^{\alpha}=\sum_{i=x, y, z} v_{i}^{\alpha} \sigma_{i}$. In Supplemental Material, Sec. E [32], we compare the currents Eqs. (5)-(7) with perturbation theory and show that Eq. (5) and Eq. (7) recover the resonant behaviour of the shift and injection currents respectively, whereas Eq. (6) becomes the nonresonant component of the shift current in the limit $e \mathbf{E}\langle c|\mathbf{r}| v\rangle \ll \Gamma$.

Now, to analyze the clean limit behavior of the injection current Eq. (7), it is useful to take the approximation in which both the diagonal $h_{z}$ matrix elements in Eq. (2) are greater than $\Gamma$ and the off-diagonal elements $h_{x, y}$. This is typically well satisfied in most solids except in special situations such as resonant absorption on extremely flat bands, and we will demonstrate that this is a good approximation by explicit calculations later on. Therefore we replace $1 /\left(h^{2}+\Gamma^{2} / 4\right) \approx \pi \delta\left(h_{z}\right) / \sqrt{h_{x}^{2}+h_{y}^{2}+\Gamma^{2} / 4}$, which leads to the following expression:

$$
\begin{aligned}
J_{3}^{\alpha}= & \pi \frac{e}{\hbar} \int \frac{d \mathbf{k}}{(2 \pi)^{3}}\left(f_{2}-f_{1}\right)\left(v_{1}^{\alpha}-v_{2}^{\alpha}\right) \\
& \times \frac{\left|\frac{e \mathbf{E}}{\hbar \omega} \cdot\left(\frac{\partial H_{0}(\mathbf{k})}{\partial \mathbf{k}}\right)_{12}\right|^{2}}{\sqrt{\left|\frac{e \mathbf{E}}{\hbar \omega} \cdot\left(\frac{\partial H_{0}(\mathbf{k})}{\partial \mathbf{k}}\right)_{12}\right|^{2}+\frac{\Gamma^{2}}{4}}} \delta\left(\epsilon_{1}-\epsilon_{2}+\hbar \omega\right),
\end{aligned}
$$

where $\mathbf{v}_{1,2}$ are conduction and valence band velocities respectively. We again see that in the limit of fast relaxation $(e \mathbf{E} \cdot\langle c|\mathbf{r}| v\rangle \ll \Gamma)$ Eq. (8) reproduces the behaviour predicted by perturbation theory (see Supplemental Material, Sec. A [32]). Remarkably, however, in the clean limit $(\Gamma \rightarrow 0)$, the above formula predicts a finite current, in sharp contrast to the naive extrapolation of perturbative result. In other words, the relaxation rate in the denominator of the perturbative expressions acquires a nonperturbative modification by the driving electric field of the form:

$$
\frac{1}{\Gamma} \rightarrow \frac{1}{\sqrt{4\left|\frac{e \mathbf{E}}{\hbar \omega} \cdot\left(\frac{\partial H_{0}(\mathbf{k})}{\partial \mathbf{k}}\right)_{12}\right|^{2}+\Gamma^{2}}} .
$$

Therefore in the clean limit, the injection current scales as the absolute value of the electric field, $\mathbf{J}_{3} \propto|\mathbf{E}|$, and, accordingly, it is proportional to the square root of the radiation intensity. On the other hand, the term $\mathbf{J}_{1}$ from Eq. (5), which reduces to the usual resonant shift current from perturbation theory (see Supplemental Material, Sec. A [32] for details), can be seen to vanish in the clean limit $\Gamma \rightarrow 0$ from Eq. (5). This is noteworthy because in the perturbative regime $(e \mathbf{E}\langle c|\mathbf{r}| v\rangle \ll \Gamma)$ the shift current naively approaches a finite value in the $\Gamma \rightarrow 0$ limit.

Synchronization and Rabi limit of rectification.-While the Keldysh formalism allows for a description with arbitrary strength of coupling to the bath, there is a simpler way to understand the ideal behavior in the limit of vanishing coupling to the bath $(\Gamma \rightarrow 0)$. In fact, this limit can be understood simply as a form of Rabi oscillations associated with the interband transitions driven by the oscillating field. We will describe how to understand this limit within the picture of the periodic Gibbs ensemble (PGE) [40,48-50] that captures the steady state synchronization of the system with the driving field.

Consider an initial state described by a density matrix $\rho_{0}$. This density matrix can be decomposed in the eigenstates of the time dependent Hamiltonian, $\psi_{\alpha}(t)$, and therefore the state at any later time $t$, is given by:

$$
\rho(t)=\sum_{\alpha \beta} \rho_{\alpha \beta} \psi_{\alpha}(t) \psi_{\beta}^{\dagger}(t)
$$

where $\rho_{\alpha \beta}=\operatorname{Tr}\left[\rho_{0} \psi_{\alpha}\left(t_{0}\right) \psi_{\beta}^{\dagger}\left(t_{0}\right)\right]$. Now, the Floquet theorem implies that, barring accidental degeneracies, the operators $\psi_{\alpha}(t) \psi_{\beta}(t)^{\dagger}$ are only periodic when $\alpha=\beta$. The late-time synchronization associated with the PGE can be understood as a process in which the memory of these off-diagonal amplitudes of the density matrix in the Floquet basis disappears in a kind of thermalization process, leading to a steady state that is exactly periodic and synchronized with the drive (see Supplemental Material, Sec. G [32]):

$$
\rho_{\mathbf{P G E}}=\sum_{\alpha} \rho_{\alpha \alpha} \psi_{\alpha}(t) \psi_{\alpha}^{\dagger}(t)
$$


Remarkably the above ensemble is identical to the one that we have obtained within the Keldysh formalism in the limit of $\Gamma \rightarrow 0$, when one chooses the initial state $\rho_{0}$ to be the equilibrium Fermi-Dirac density matrix in the absence of the periodic perturbation, with the chemical potential and the temperature of the bath (see Supplemental Material, Sec. E [32] for more details). In fact, within the same rotating-wave approximation used to solve the Floquet problem, this density matrix is explicitly given by (see Supplemental Material, Sec. G [32]):

$$
\hat{\rho}_{\mathbf{P G E}}(t)=\left(\begin{array}{cc}
f_{2} & 0 \\
0 & f_{1}
\end{array}\right)+\frac{f_{1}-f_{2}}{2 h^{2}}\left(\begin{array}{cc}
h_{x}^{2}+h_{y}^{2} & h_{z} h_{+} e^{-i \omega t} \\
h_{z} h_{-} e^{i \omega t} & -h_{x}^{2}-h_{y}^{2}
\end{array}\right) .
$$

This density matrix encodes the physics of Rabi oscillations (see Supplemental Material, Sec. F [32] for details). The above reduces exactly to the density matrix in Eq. (4) in the clean limit $\Gamma \rightarrow 0$, once it is expressed in the Floquet picture [see Eq. (3)], and therefore predicts the same rectification currents that we have previously described in the clean limit.

We would like to note that most studies of PGE to date have focused on what might be called internal synchronization, which considers a closed system acting as its own bath. In this context, the initial condition, $\rho_{0}$, is freely chosen and it is not unique. In our context, however, the emergence of the PGE follows from different principles. Coupled to the bath, the system loses memory of its initial state at late times. It does so by flowing towards a unique stable periodic solution. Remarkably, in the limit of weak coupling to the bath, this steady state coincides exactly with one specifically chosen PGE, whose initial condition is the one associated with the thermal equilibrium system and with an infinitesimal coupling to the bath in the absence of the periodic drive.

Therefore, although we have performed our calculations in a rather specific microscopic setting, we have been able to recover the universality of the PGE in the limit of weak coupling to the bath that we are using. Since the PGE can be justified under generalized entropy maximixation principles $[40,48-50]$, this is a compelling indication that our results describe the behavior of a large class of systems coupled to ideal heat baths.

Photocurrents for 3D Weyl and 2D Dirac fermions.One important distinction between shift and injection currents is their transformations under time-reversal (TR) symmetry [51,52]. The shift current can exist in TR invariant materials illuminated with linearly polarized light, whereas the injection current requires breaking of TR symmetry, namely either by shining linearly polarized light on a TR broken material $[53,54]$, or by shining circularly polarized light, also known as the circular-photogalvanic effect, which has an interesting manifestation in Weyl semimetals [16,18,31,55-65].

We would like to illustrate this behavior for representative nodal fermions with Hamiltonians that are linear in momentum. These linear in $\mathbf{k}$ Hamiltonians have negligible shift currents (see details in Supplemental Material, Sec. J [32]) and therefore allow us to focus on the behavior of injection currents, which we will consider in this section. We will consider two types of model that are relevant to a large class of materials. The first is an ideal threedimensional Weyl fermion, and our focus will be on the nonperturbative modifications to the CPGE. As we will see, our results are in perfect agreement with those obtained recently in Ref. [30]. The second will be a twodimensional-tilted Dirac massive fermion, and our focus will be to investigate the nonperturbative regime of rectification for linearly polarized light in a time reversal breaking system.

The ideal three-dimensional Weyl Hamiltonian is

$$
\hat{H}_{0}=v_{0} \sum_{\alpha=x, y, z} k_{\alpha} \cdot \hat{\sigma}_{\alpha} .
$$

Here $v_{0}$ is a Fermi velocity and $\hat{\sigma}_{\alpha}$ are Pauli matrices. This model respects TR but breaks inversion symmetry. When the system has a finite chemical potential, light absorption occurs above a threshold frequency $\hbar \omega>2 \epsilon_{F}$ [see Fig. 2(a)]. By using the formula from Eq. (8), one obtains the following nonperturbative approximate expression of the injection current above such a threshold (see Supplemental Material, Sec. I [32] for details):

$\mathbf{J}_{3} \approx \frac{i \pi^{2} e^{2} \omega}{v_{0}(2 \pi)^{3}} \frac{\left[\mathbf{E}^{*} \times \mathbf{E}\right]}{60 \sqrt{|\mathbf{E}|^{2}+\frac{\Gamma^{2} \hbar^{2} \omega^{2}}{4 v_{0}^{2} e^{2}}}} \frac{12|\mathbf{E}|^{2}+5 \frac{\Gamma^{2} \hbar^{2} \omega^{2}}{v_{0}^{2} e^{2}}}{|\mathbf{E}|^{2}+\frac{\Gamma^{2} \hbar^{2} \omega^{2}}{4 v_{0}^{2} e^{2}}}$,

where $|\mathbf{E}|^{2}=\mathbf{E}^{*} \cdot \mathbf{E}$, with $\mathbf{E}$ understood as the complex vector defined in Eq. (1) [see Supplemental Material, Sec. I [32] for comparison of this approximate formula against direct evaluation from the integral in Eq. (8)]. Equation (14) in the perturbative regime $\left(e^{2} v_{0}^{2}|\mathbf{E}|^{2} \ll \Gamma^{2} \hbar^{2} \omega^{2}\right)$ approaches the known result $[16,18] \mathbf{J}_{3} \approx \hbar \beta\left[\mathbf{E}^{*} \times \mathbf{E}\right] / \Gamma$, where $\beta=$ $i \pi e^{3} /\left(3 h^{2}\right)$. However, in the Rabi regime $\left(e^{2} v_{0}^{2}|\mathbf{E}|^{2} \gg\right.$ $\left.\Gamma^{2} \hbar^{2} \omega^{2}\right)$ interestingly, the injection current approaches a value that is independent of the relaxation rate and is given by:

$$
\mathbf{J}_{3} \approx \zeta \frac{\beta \hbar^{2} \omega}{e v_{0}|\mathbf{E}|}\left[\mathbf{E}^{*} \times \mathbf{E}\right]
$$

Here $\zeta \approx 0.3$ is a numerical prefactor with a weak dependence on the degree of light polarization. Its value for perfectly circularly polarized light can be computed exactly from Eq. (8) to be $\zeta=1 /(2 \sqrt{2})$, in agreement with Ref. [30] (see Supplemental Material, Sec. I [32] for details). 
3D Weyl Fermions (circular light)

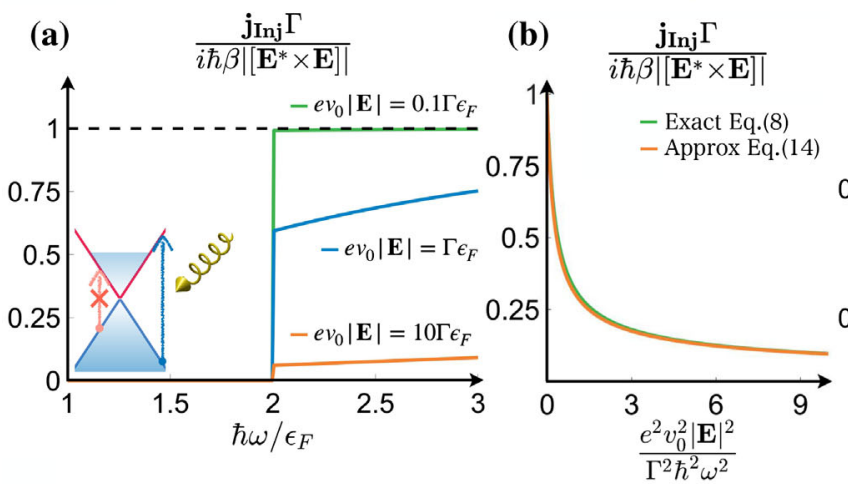

2D Dirac Fermions (linear light)
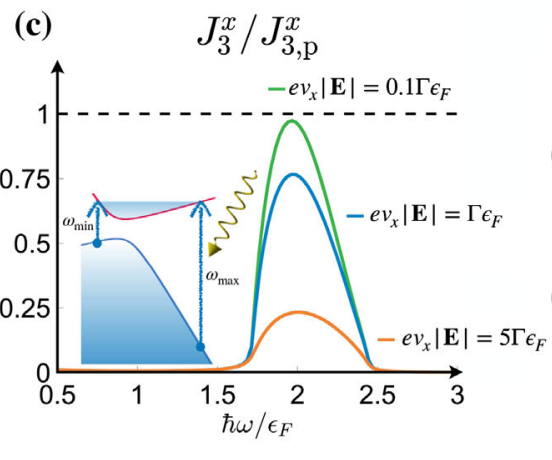

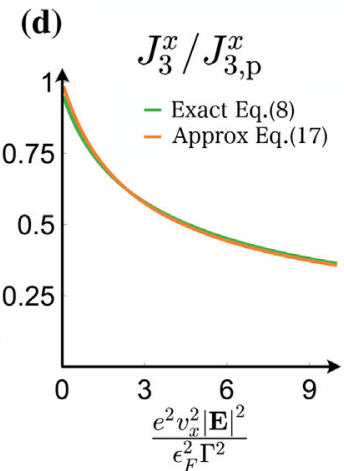

FIG. 2. (a) Rectified current dependence on frequency, and (b) on electric field amplitude for 3D Weyl fermion $[\mathbf{E} /|\mathbf{E}|=(0, i \sin \pi / 8 \sin \pi / 4, \cos \pi / 4+i \cos \pi / 8 \sin \pi / 4)]$, (c) Rectified current dependence on frequency, and (d) on electric field amplitude for 2D Dirac fermion $\left[\mathbf{E} /|\mathbf{E}|=(1,0), u_{x} / v_{x}=0.2, m=0.5 \epsilon_{F}\right]$.

The behavior of the rectified current in these two regimes and their crossovers are shown in Figs. 2(a) and 2(b).

We will now consider a two-dimensional Dirac Hamiltonian given by:

$$
\hat{H}=u_{x} k_{x} \hat{\mathbb{1}}+v_{x} k_{x} \hat{\sigma}_{x}+v_{y} k_{y} \hat{\sigma}_{y}+m \hat{\sigma}_{z},
$$

where $m$ is the mass which breaks time-reversal symmetry, $v_{x}, v_{y}$ are anisotropic Fermi velocities, and $u_{x}>0$ is the tilt term that breaks inversion. The above model features absorbtion within a window of frequency given by $\left(2 \epsilon_{F}-2 \alpha \sqrt{\epsilon_{F}^{2}-m^{2}+\alpha^{2} m^{2}}\right) /\left(1-\alpha^{2}\right)<\hbar \omega<\left(2 \epsilon_{F}+\right.$ $\left.2 \alpha \sqrt{\epsilon_{F}^{2}-m^{2}+\alpha^{2} m^{2}}\right) /\left(1-\alpha^{2}\right)$ [see Fig. 2(c)]. In this window the maximum current occurs when $\hbar \omega \approx 2 \epsilon_{F}$ [see Fig. 2(c)], and for the electric field along the tilt direction. From Eq. (8), the corresponding component of the injection current can be approximated as (see Supplemental Material, Sec. I [32]):

$$
\begin{aligned}
J_{3, \max }^{x} \approx & \frac{e^{2} v_{x}}{\hbar v_{y}} \frac{\sqrt{\epsilon_{F}^{2}-m^{2}}}{60 \pi} \frac{\frac{|\mathbf{E}|^{2}}{\epsilon_{F}^{2}}}{\sqrt{\frac{|\mathbf{E}|^{2}}{\epsilon_{F}^{2}}+\frac{\Gamma^{2}}{e^{2} v_{x}^{2}}}} \\
& \times \frac{5 \frac{\Gamma^{2}}{e^{2} v_{x}^{2}}\left(1+2 \frac{m^{2}}{\epsilon_{F}^{2}}\right)+\frac{|\mathbf{E}|^{2}}{\epsilon_{F}^{2}}\left(6+13 \frac{m^{2}}{\epsilon_{F}^{2}}-4 \frac{m^{4}}{\epsilon_{F}^{4}}\right)}{\frac{|\mathbf{E}|^{2}}{\epsilon_{F}^{2}}+\frac{\Gamma^{2}}{e^{2} v_{x}^{2}}} .
\end{aligned}
$$

Within perturbation theory this current would be $J_{3, p}^{x}=$ $e^{3} v_{x}^{2} \sqrt{\epsilon_{F}^{2}-m^{2}}\left(1+2 m^{2} / \epsilon_{F}^{2}\right)|\mathbf{E}|^{2} /\left(12 \pi \hbar v_{y} \Gamma \epsilon_{F}^{2}\right)$. We use $J_{3, p}^{x}$ to normalize the numerical nonpertubative results shown in Figs. 2(c) and 2(d), so that deviations from 1 signal deviations from the perturbative regime.

Summary and experimental outlook.-We have developed a formalism which captures on equal footing the perturbative regime of fast relaxation $(e \mathbf{E}\langle c|\mathbf{r}| v\rangle \ll \Gamma)$ and the nonperturbative regime of strong light intensity $(e \mathbf{E}\langle c|\mathbf{r}| v\rangle \gg \Gamma)$ of current rectification for interband transitions. In the perturbative regime, we recover the well-known behavior according to which shift currents approach a value that is independent of the relaxation rate $\Gamma$, while injection currents scale as $1 / \Gamma$. Interestingly in the opposite nonperturbative clean limit of slow relaxation $(\Gamma \rightarrow 0)$ the shift current vanishes, while the injection current approaches a finite value independent of $\Gamma$, but with a net current that scales as the square root of the radiation intensity, which can guide its identification in experiments. We have shown that this nonperturbative clean limit can be understood as optical Rabi oscillations synchronized with the incident radiation that realizes a time dependent generalized periodic Gibbs ensemble in a setting very different from its initial proposal.

Nodal Weyl semimetals are promising platforms to realize the Rabi regime because their interband dipole matrix element diverges when approaching the Weyl node as $\langle c|\mathbf{r}| v\rangle \propto 1 / k$. As a consequence, they can access this nonperturbative regime above a light intensity that decreases with frequency, namely when $e v_{0}|\mathbf{E}|>\hbar \Gamma \omega$. For RhSi [66,67], using $\hbar \Gamma^{-1} \approx 10$ ps [59] we estimate that the nonperturbative Rabi regime will be accessed at light intensities above $4 \times 10^{5} \mathrm{~W} / \mathrm{cm}^{2}$ for a photon energy of $\hbar \omega \approx 0.5 \mathrm{eV}$, but this required light intensity can be decreased as $\omega^{2}$ at lower photon energies.

We wish to thank Achilleas Lazarides, Kin Fai Mak, and Andrea Cavalleri for valuable discussions and correspondence, and Nikita Leppenen and Leonid Golub for sharing the calculations that helped us understand the precise connection to their work in Ref. [30]. We acknowledge financial support from the Deutsche Forschungsgemeinschaft through SFB 1143 (Project No. 247310070) and Cluster of Excellence ct.qmat (EXC 2147, Project No. 39085490). 
[1] V. I. Belinicher and B. I. Sturman, Sov. Phys. Usp. 23, 199 (1980).

[2] R. von Baltz, Ferroelectrics 35, 131 (1981).

[3] B. I. Sturman and V. M. Fridkin, Photovoltaic and Photorefractive Effects in Noncentrosymmetric Materials (CRC Press, Philadelphia, 1992).

[4] W. Kraut and R. von Baltz, Phys. Rev. B 19, 1548 (1979).

[5] R. von Baltz and W. Kraut, Phys. Rev. B 23, 5590 (1981).

[6] V. Belinicher, E. Ivchenko, and B. Sturman, Zh. Eksp. Teor. Fiz. 83, 649 (1982).

[7] C. Aversa and J. E. Sipe, Phys. Rev. B 52, 14636 (1995).

[8] J. E. Sipe and A. I. Shkrebtii, Phys. Rev. B 61, 5337 (2000).

[9] B. I. Sturman, Phys. Usp. 63, 407 (2020).

[10] S. M. Young and A. M. Rappe, Phys. Rev. Lett. 109, 116601 (2012).

[11] I. Sodemann and L. Fu, Phys. Rev. Lett. 115, 216806 (2015).

[12] T. Morimoto and N. Nagaosa, Sci. Adv. 2, e1501524 (2016).

[13] N. Nagaosa and T. Morimoto, Adv. Mater. 29, 1603345 (2017).

[14] O. Matsyshyn and I. Sodemann, Phys. Rev. Lett. 123, 246602 (2019).

[15] D. E. Parker, T. Morimoto, J. Orenstein, and J. E. Moore, Phys. Rev. B 99, 045121 (2019).

[16] F. de Juan, A. G. Grushin, T. Morimoto, and J. E. Moore, Nat. Commun. 8, 15995 (2017).

[17] O. Matsyshyn et al., J. Phys. D 54, 404001 (2021).

[18] C. Chan, N. H. Lindner, G. Refael, and P. A. Lee, Phys. Rev. B 95, 041104 (2017).

[19] D. Vanderbilt, Berry Phases in Electronic Structure Theory: Electric Polarization, Orbital Magnetization and Topological Insulators (Cambridge University Press, Cambridge, England, 2018).

[20] J. E. Moore and J. Orenstein, Phys. Rev. Lett. 105, 026805 (2010)

[21] K. Kang, T. Li, E. Sohn, J. Shan, and K. F. Mak, Nat. Mater. 18, 324 (2019).

[22] Q. Ma, S. Xu, H. Shen, D. MacNeill, V. Fatemi, T. Chang, A. M. Mier Valdivia, S. Wu, Z. Du, C. Hsu et al., Nature (London) 565, 337 (2019).

[23] J. A. Brehm, S. M. Young, F. Zheng, and A. M. Rappe, J. Chem. Phys. 141, 204704 (2014).

[24] T. Rangel, B. M. Fregoso, B. S. Mendoza, T. Morimoto, J. E. Moore, and J. B. Neaton, Phys. Rev. Lett. 119, 067402 (2017).

[25] A. M. Cook, B. M. Fregoso, F. de Juan, S. Coh, and J. E. Moore, Nat. Commun. 8, 14176 (2017).

[26] T. Morimoto, M. Nakamura, M. Kawasaki, and N. Nagaosa, Phys. Rev. Lett. 121, 267401 (2018).

[27] D. Kumar, C.-H. Hsu, R. Sharma, T.-R. Chang, P. Yu, J. Wang, G. Eda, G. Liang, and H. Yang, Nat. Nanotechnol. 16, 421 (2021).

[28] S. Kitamura, N. Nagaosa, and T. Morimoto, Phys. Rev. B 102, 245141 (2020).

[29] V. V. Afonin, V. L. Gurevich, and R. Laiho, Phys. Rev. B 52, 2090 (1995).

[30] N. V. Leppenen, E. L. Ivchenko, and L. E. Golub, Phys. Status Solidi (b) 256, 1900305 (2019).

[31] R. M. A. Dantas, Z. Wang, P. Surwka, and T. Oka, Phys. Rev. B 103, L201105 (2021).
[32] See Supplemental Material at http://link.aps.org/ supplemental/10.1103/PhysRevLett.127.126604 for details on: (a) perturbative approach, (b)-(e) Floquet and Keldysh formalisms, (f)-(g) Rabi limit and periodic Gibbs ensemble, (i)-(j) Rectifcation for Weyl and Dirac fermions.

[33] R. B. James and D. L. Smith, Phys. Rev. Lett. 42, 1495 (1979).

[34] R. B. James and D. L. Smith, Phys. Rev. B 21, 3502 (1980).

[35] D. Parshin and A. Shabaev, Zh. Eksp. Teor. Fiz. 92, 1471 (1987).

[36] L. Gerchikov, D. Parshin, and A. Shabayev, Zh. Eksp. Teor. Fiz. 96, 1046 (1989).

[37] T. Oka and H. Aoki, Phys. Rev. B 79, 081406 (2009).

[38] A.-P. Jauho, N. S. Wingreen, and Y. Meir, Phys. Rev. B 50, 5528 (1994).

[39] A. Kamenev, Field Theory of Non-Equilibrium Systems (Cambridge University Press, Cambridge, England, 2011).

[40] A. Lazarides, A. Das, and R. Moessner, Phys. Rev. Lett. 112, 150401 (2014).

[41] A. Russomanno, A. Silva, and G. E. Santoro, Phys. Rev. Lett. 109, 257201 (2012).

[42] B. M. Fregoso, Y. H. Wang, N. Gedik, and V. Galitski, Phys. Rev. B 88, 155129 (2013).

[43] A. Kamenev, arXiv:cond-mat/0412296.

[44] K. Johnsen and A.-P. Jauho, Phys. Rev. Lett. 83, 1207 (1999).

[45] A.-P. Jauho, N. S. Wingreen, and Y. Meir, Phys. Rev. B 50, 5528 (1994).

[46] S. Kohler, J. Lehmann, and P. Hanggi, Phys. Rep. 406, 379 (2005).

[47] T. Kitagawa, T. Oka, A. Brataas, L. Fu, and E. Demler, Phys. Rev. B 84, 235108 (2011).

[48] V. Khemani, A. Lazarides, R. Moessner, and S. L. Sondhi, Phys. Rev. Lett. 116, 250401 (2016).

[49] A. Lazarides, A. Das, and R. Moessner, Phys. Rev. E 90, 012110 (2014).

[50] A. Lazarides, A. Das, and R. Moessner, Phys. Rev. Lett. 115, 030402 (2015).

[51] J. Ahn, G.-Y. Guo, and N. Nagaosa, Phys. Rev. X 10, 041041 (2020).

[52] D. Hornung and R. von Baltz, Phys. Rev. B 103, 195203 (2021).

[53] E. L. Ivchenko and G. E. Pikus, JETP Lett. 27, 604 (1978).

[54] Y. Zhang, T. Holder, H. Ishizuka, F. de Juan, N. Nagaosa, C. Felser, and B. Yan, Nat. Commun. 10, 3783 (2019).

[55] L. E. Golub and E. L. Ivchenko, Phys. Rev. B 98, 075305 (2018).

[56] F. Flicker, F. de Juan, B. Bradlyn, T. Morimoto, M. G. Vergniory, and A. G. Grushin, Phys. Rev. B 98, 155145 (2018).

[57] F. de Juan, Y. Zhang, T. Morimoto, Y. Sun, J. E. Moore, and A. G. Grushin, Phys. Rev. Research 2, 012017 (2020).

[58] A. Avdoshkin, V. Kozii, and J. E. Moore, Phys. Rev. Lett. 124, 196603 (2020).

[59] D. Rees, K. Manna, B. Lu, T. Morimoto, H. Borrmann, C. Felser, J. E. Moore, D. H. Torchinsky, and J. Orenstein, Sci. Adv. 6, eaba0509 (2020).

[60] Z. Ni, K. Wang, Y. Zhang, O. Pozo, B. Xu, X. Han, K. Manna, J. Paglione, C. Felser, A. G. Grushin et al., Nat. Commun. 12, 154 (2021).

[61] Z. Ni, K. Wang, Y. Zhang, O. Pozo, B. Xu, X. Han, K. Manna, J. Paglione, C. Felser, A. G. Grushin et al., Nat. Commun. 12, 154 (2021). 
[62] Q. Ma, S. Xu, C. Chan, C. Zhang, G. Chang, Y. Lin, W. Xie, T. Palacios, H. Lin, S. Jia et al., Nat. Phys. 13, 842 (2017).

[63] N. Nagaosa, T. Morimoto, and Y. Tokura, Nat. Rev. Mater. 5, 621 (2020).

[64] K. Sun, S.-S. Sun, L.-L. Wei, C. Guo, H.-F. Tian, G.-F. Chen, H.-X. Yang, and J.-Q. Li, Chin. Phys. Lett. 34, 117203 (2017).
[65] Z. Ji, G. Liu, Z. Addison, W. Liu, P. Yu, H. Gao, Z. Liu, A. M. Rappe, C. L. Kane, E. J. Mele et al., Nat. Mater. 18, 955 (2019).

[66] G. Chang, S.-Y. Xu, B. J. Wieder, D. S. Sanchez, S.-M. Huang, I. Belopolski, T.-R. Chang, S. Zhang, A. Bansil, H. Lin et al., Phys. Rev. Lett. 119, 206401 (2017).

[67] P. Tang, Q. Zhou, and S.-C. Zhang, Phys. Rev. Lett. 119, 206402 (2017). 